\title{
Simulation of Micro-Channel Heat Sinks for Optoelectronic Microsystems
}

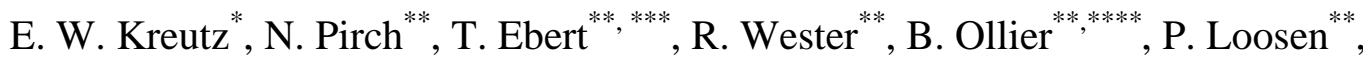 \\ R. Poprawe ${ }^{* * * *}$ \\ * Lehrstuhl für Lasertechnik, Rheinisch-Westfälische Technische Hochschule Aachen \\ Steinbachstraße 15, D-52074 Aachen, Germany, kreutz@llt-rwth.aachen.de \\ * Fraunhofer-Institut für Lasertechnik \\ Steinbachstraße 15, D-52074 Aachen, Germany \\ *** present address: PROLAS Produktionslaser $\mathrm{GmbH}$ \\ Steinbachstraße 15, D-52074 Aachen, Germany \\ **** present address: Johnsons Controls GmbH \\ Industriestraße 20 - 30, D-51399 Burscheid, Germany
}

\begin{abstract}
Water-cooled heat sinks are investigated both experimentally and theoretically as model systems to simulate the energy and mass transport in devices used for cooling of diode lasers. The design of the micro-channel heat sinks yields in a decrease of their thermal resistance and of the pressure loss of the coolant allowing to increase the output power of the diode lasers.
\end{abstract}

Keywords: micro-channel heat sink, laser diode, micro cooling system, optoelectronic microsystem

\section{INTRODUCTION}

Microsystem technology is driven by specific requirements such as the high integration density of the single elements, the three-dimensional integration of the elements in a hybrid system, the use of new materials having specific properties, as well as the overall miniaturization of the systems. This trend is closely related to another technological revolution involving optoelectronic integration. Materials science and advanced fabrication methods such as molecular-beam epitaxy, metal-organic chemical vapour deposition, and focused-ion-beam micromachining have enabled optical, electrooptical, and electronic components to be consolidated into monolithic structures. Integration of these normally disparate elements allows designers to combine the advantages of each in a single device.

The proximity of such widely desperate elements on a single structure of wafer often leads to intractable incompatibilities between materials. Interference or crosstalk between various elements also can hamper the performance of the final device.
A number of successful strategies has been developed to deal with the main problems within a highly integrated circuit, system, or device to control the flow of masses, charges, phonons and photons including mainly channels, waveguides, and substrates. As a demonstrator for microsystem simulation the temperature and flow field as well in water-cooled heat sinks for packaging of hybrid systems is investigated both experimentally and theoretically.

The water-cooled heat sink is an excellent model system to simulate the energy and mass transport in cooling devices for diode lasers supporting its future development and dissemination in production technology. One crucial problem for the further development of diode lasers to higher power densities is the effective carrying-off of the heat resulting from the conversion process in order to minimize the resulting thermal load and thereby thermal strain and stress in the optoelectronic microsystem diode laser [1]. Due to the high conversion efficiency of electrical to optical energy diode lasers have emerged as sources either for pumping of materials for generation of laser radiation or for direct use in processing of materials. High output powers of the diode lasers require high electric excitation currents originating in a high thermal load.

In order to be used for cooling of diode lasers water cooled micro-channel copper heat sinks [2] are investigated as model systems to simulate the energy and mass transport within optoelectronic microsystems. Copper is superior in thermophysical properties and costs to silicon and glass also used as micro-channel heat sinks [3], making copper a hopeful candidate as reasonable and effective material for heat sinks [2]. The main emphasis of this paper is on the pressure loss and the thermal resistance both of them influencing the lifetime and the costs of the heat sinks. 


\section{COPPER HEAT SINKS}

Figure 1 shows the copper heat sink under investigation including the mounting of the diode laser bar. Each heat sink consists of five copper slices (Fig. 1), which are manufactured by cutting with laser radiation and joined by diffusion welding. The bottom slice (1) of the heat sink contains the coolant in- and outlet, the middle slices $(2-4)$ represent the micro cooling device with the in (2)- and outlet (4) manifold of the coolant and the micro-structured cooler (3) and the top slice (5) acts as sealing cover and mounting plane for the diode laser bar (Fig. 1). In case of diode laser stacks the slices (1) and (5) are identical to provide the cooling allover the single diode bars assembled. Further details including properties and mounting of the cooler have been reported elsewhere [2, 4].
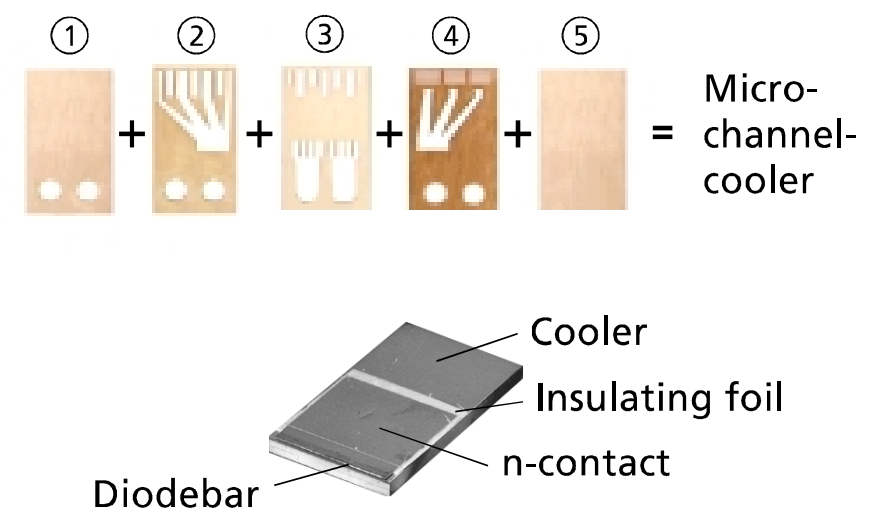

Figure 1: Design of sliced copper heat sink (above) and mounted diode laser on top of it (below)

These components, including diode laser bar with $40 \mathrm{~W} \mathrm{cw}$ output power mounted on top of the copper heat sink, usually are operated with the following operational parameters water flow rate of $500 \mathrm{ml} / \mathrm{min}$, pressure loss of $5 \mathrm{bar}$, approximated thermal load of $60 \mathrm{~W}$, approximated junction temperature rise of $27 \mathrm{~K}$, and a thermal resistance of $0.44 \mathrm{~K} / \mathrm{W}$ based on a 10 $\mathrm{x} 0.6 \mathrm{~mm}^{2}$ contact area.

\section{PRESSURE LOSS}

For a detailed analysis of heat and mass flow, the flow and pressure loss in every part of the heat sinks have to be determined. The corresponding experimental investigations have been reported elsewhere [4] with the geometric and dynamic similarity shown in order to prove the validation of the model results.

The whole micro-channel cooling device may be subdivided into two areas, the in- and outlet manifold and the micro structured cooling part. The analysis showed that less than 10 $\%$ of the pressure loss is caused by the micro channels. The remaining $90 \%$ pressure loss has been attributed to the following processes in the in- and outlet manifold:

- friction losses

$13 \%$

- abrupt changes of flow cross-sections $26 \%$

- hard edged flow direction changes $61 \%$

The following measures have been taken in order to reduce pressure losses as well as thermal resistance:

- matching of the different flow cross-sections by appropriate diffusor structures

- reduction of the number of hard edged flow direction changes by simplifications of the manifold structures

- increase of the flow rate by increasing the total height of the heat sinks

The results may be used to adapt the geometry of any channel system and the fluid flow for effective carrying-off of heat within any optoelectronic microsystem.

\section{DESIGN OF MICRO COOLERS}

Due to the design of the copper heat sinks (Fig. 1), it is not possible to control the flow of heat by the coolant in an optimum way. The passage of the coolant between the slices (2) to (4) within the micro-channel cooler follows either sharp edges in the free cross-section or abrupt changes in the crosssection. Following the measures of experimental investigations ([4], see above) the necessary changes of flow direction by one big step within the micro-channel cooler were effected by implementation of several little steps [5] to avoid the unavoidable draw-back responsible for the pressure losses. The little steps create whirling rolls, so that the coolant will be led by these rolls and not by the boundary edges of the several slices. If the micro-channel coolers can be built higher, the increased number of slices allows an increasing number of steps. Therefore, a more sensitive design of the step structure will be possible. The cross section course of the coolant flow of a $1,5 \mathrm{~mm}$ high standard and a $1,5 \mathrm{~mm}$ high optimized micro cooler is shown in figure 2 for comparison. The corresponding pressure losses versus coolant flow is shown in figure 3 for various copper micro-channel coolers.

To optimize the thermal properties of the micro-channel coolers, the orientation of the cooling structure may be changed (Fig. 4). In the standard cooling devices the micro structure was carried out as micro channels. In the improved micro coolers the orientation of the cooling structure was shifted by $90^{\circ}$, so that the water supply is in the same layer as the micro structure. In this micro cooling structure the heat spreading effect is not limited to the copper layer on top of the heat sink, the heat spreading effect extends to the micro structure (micro fins) itself [6]. A third way to carry out a cooling structure are little bars with a cross section of $100 \mu \mathrm{m}$ 
$\mathrm{x} 100 \mu \mathrm{m}$ and a height of $400 \mu \mathrm{m}$ (micro bars). The achieved numerical values of thermal resistance are summarized in table 1 for the various structures. The increasing heat transfer from the copper to the coolant is caused by the origin of whirls in the micro structure. On the one hand whirls occur due to the rise of flow velocity (micro fins), on the other hand whirls will be built up because of the special layout of the structures (micro bars).
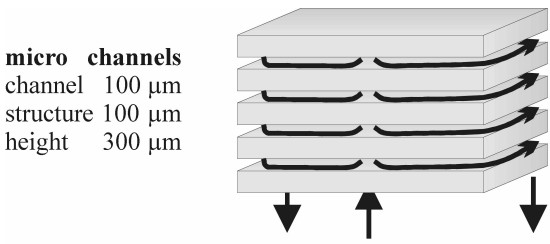

m i c ro fi n s

channel $100 \mu \mathrm{m}$

fins $\quad 100 \mu \mathrm{m}$

height $\quad 300 \mu \mathrm{m}$

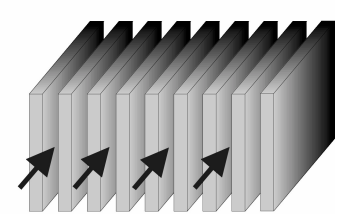

m i c r o b a r s

edges $100 \mu \mathrm{m}$

distance $100 \mu \mathrm{m}$

height $400 \mu \mathrm{m}$

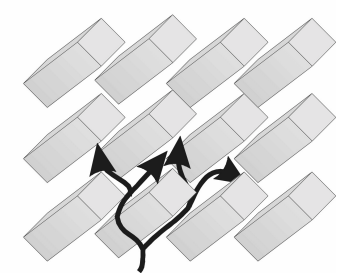

Figure 4: Design of different structures for micro coolers

\section{CONCLUSION}

The integration of micro coolers enables an effective carrying-off of the conversion heat in optoelectronic systems such as high power diode lasers. The amount of heat which is carried-off per time depends on the thermophysical properties of the coolant and the flow dynamics in the structure of the micro coolers. The fluid is driven by the pressure loss applied at the in- and outlet manifold of the micro channels depending on the design of the structure of the micro coolers.

The optimized structure of micro cooler heat sinks give the choice of either sticking to a flow rate of $500 \mathrm{ml} / \mathrm{min}$ and take advantage of the ten times lower pressure loss at an improved thermal resistance of $0,39 \mathrm{k} / \mathrm{W}$, or taking into account a pressure loss of 4 bar and make use of the higher flow rate of $1200 \mathrm{ml} / \mathrm{min}$, which leads to a decreased thermal resistance of $0,29 \mathrm{~K} / \mathrm{W}$. Based on this reduced thermal resistance and a maximum junction temperature typically a cw laser output power of more than $60 \mathrm{~W}$ can be achieved. In contrast, a cW output power of $116 \mathrm{~W}$ can been achieved during a test period of several minutes.

\section{ACKNOWLEDGEMENT}

The funding by the Bundesministerium für Bildung, Wissenschaft, Forschung und Technologie under contract no. 13 N6501 is gratefully acknowledged.

\section{REFERENCES}

[1] P. Loosen, H.-G. Treusch, C. R. Haas, U. Gardenier, M. Weck, V. Sinhoff, St. Kasperowski, R. vor dem Esche, SPIE Proc. Series 2382, 78, 1995

[2] T. Ebert, thesis, RWTH Aachen, 1998.

[3] R. Beach, IEEE J. Quant. Electr. 28, 966, 1992.

[4] T. Ebert, J. Biesenbach, H.-G. Treusch, P. Loosen, R. Poprawe, SPIE Proc. Series 3097, 578, 1997

[5] E. Tuckenbrodt, Fluidmechanik I, Springer Verlag, Berlin, 1980. 